\title{
Impacts of Handoff on TCP Performance in Mobile Wireless Computing
}

\author{
Aldar C.-F. Chanł’, Danny H. K. Tsangł, Sanjay Gupta§ \\ $\dagger$ Department of Electrical and Electronic Engineering, \\ The Hong Kong University of Science and Technology, Clear Water Bay, Kowloon, HONG KONG \\ \{eealdar, eetsang\}@ee.ust.hk \\ $\S$ Motorola GSM Product Division, \\ 1501 West Shure Drive (IL27-3205), Arlington Heights, IL 60004, U.S.A. \\ guptasn@cig.mot.com
}

\begin{abstract}
The effects of intermittent disconnections due to host motions on the performance of TCP connections are investigated. Fading and handoff due to host motion cause increased delay and packet losses to the active transport layer connection. TCP interprets these as signs of network congestion. As a result, it promptly throttles its transmissions and backoffs its timers, leading to slow post-handoff recovery of the transmission and long idle time. These cause severe end-to-end throughput degradation and unreasonably long interactive delay for human interaction. In this paper, we present three phenomena observed (long communication pause, slow post-handoff recovery, and successive timeouts) which are the main causes for TCP performance degradation in the presence of handoff. To alleviate these effects on TCP, two schemes, PROBE and BUFFER+FREEZE, are proposed. PROBE makes TCP aware of mobility and adapts the protocol to the mobile environment. Whereas, BUFFER+FREEZE tries to hide the effects of motion from TCP by buffering at the basestation and freezing the action of the TCP source.
\end{abstract}

\section{INTRODUCTION}

TCP (Transmission Control Protocol)[10] is a commonly used reliable transport layer protocol over a wide range of networks such as Internet and LAN (Local Area Network). It is a sliding window protocol designed for wired networks and fixed hosts. It assumes that network congestion is the sole cause of packet/segment loss and unexpected delay increase. Hence, whenever packet loss is detected, TCP will promptly reduce its transmission rate by reducing its window size (cwnd) and backoff its retransmission timer.

Users in the mobile cellular environment often carry hosts across cell boundaries without prior warning and in the midst of data transfers. As a result, when used in mobile wireless networks, TCP can experience packet losses and unexpected delay increases which are unrelated to network congestions but due to handoff.

The packets lost during handoff (due to little overlapping between the original and the new basestation) can be false congestion alarms to TCP causing it to needlessly reduce its sending rate and backoff its retransmission timer. The timer backoff causes long communication pauses and hence long idle time before transmission resumption after the completion of handoff. Besides, the small values of the window size (cwnd) and the slow-start threshold (ssthresh) after handoff causes unnecessary link under-utilization and slow recovery after handoff. These together lead to significant throughput drop.

Due to its popularity, the study of the implementations of TCP over mobile cellular networks is necessary for smooth and harmonized introduction of wireless data system. In order to alleviate the effects of handoff on TCP performance, it is necessary to either hide motion from TCP or make TCP mobile-aware to distinguish between packet losses due to network congestion and those due to mobility. Two schemes are proposed to improve the perfor- mance of TCP in the presence of handoff, namely PROBE and BUFFER+FREEZE.

PROBE uses explicit information from the network layer (Mobile IP) to signal the completion of handoff so as to reduce the long communication pause after handoff. To trigger faster retransmissions, the TCP source is informed explicitly the segments missing at the receiver by a bitmask. Besides, the TCP source in PROBE estimates the capacity of the new basestation and sets the ssthresh and cwnd accordingly in order to solve the problems caused by small post-handoff cwnd and ssthresh.

BUFFER+FREEZE buffers packets at the radio interface to minimize losses during handoff. In addition, it tries to freeze the action of the TCP source to avoid unnecessary reduction of cwnd and ssthresh through the use of the parameter, which advertizes the receiver buffer size, in the TCP header of the acknowledgments.

The remainders of the paper are as follows. In the next section, the effects of handoff on TCP are presented. Then in section III, the two schemes proposed, namely PROBE and BUFFER+FREEZE, are addressed. Performance comparison among the schemes are presented in section IV. Finally, the last section concludes the paper.

\section{The Effects of Motion}

The end-to-end TCP throughput was found to drop significantly in the presence of motion [2], [8]. Motion triggered disconnections lead to three phenomena which are the major causes of TCP throughput degradation. They are long communication pauses, slow post-handoff recovery and successive timeouts due to disconnections.

\section{A. Long Communication Pauses}

In mobile environment, when a user moves between cells and performs a handoff, there is a blackout period or disconnection ${ }^{1}$ while the BS (basestations or mobile support station) learns about the MH's (Mobile Host) motion and the new route to the MH converges. Disconnections may also be due to physical obstacles or interference from neighboring objects as the MH moves. The duration of disconnection can be fairly long, for example, the disconnection may last as long as 1 minute in the Metricom's Ricochet network[11]. Long disconnections can also occur during non-overlapping cell handoffs.

MH and BS are usually unaware of the disconnections and continue packet transmissions, resulting in the subsequent loss of TCP segments and acknowledgments. An active TCP session thus loses up to a full window's worth of packets and related acknowledgment during the disconnection. These losses of packets and acknowledgments trigger retransmission timeouts at the TCP

\footnotetext{
${ }^{1}$ In this paper, the terms "disconnection", "cell crossing" and "handoff" are used interchangeably
} 


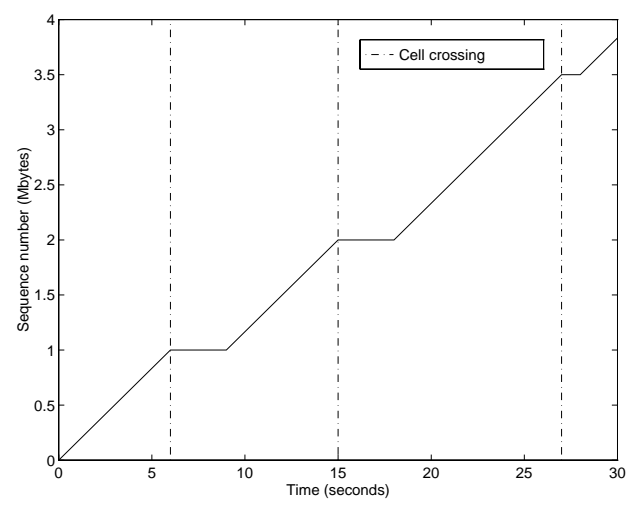

Fig. 1. Behavior of TCP sequence number in response to disconnections

sender. TCP assumes these losses as consequences of network congestion. In response, it retransmits the earliest unacknowledged packet, backoffs its timer and shrinks cwnd and ssthresh. If the disconnection has not passed, the retransmitted packets are also lost and TCP will wait for another timeout before retransmitting these packets.

One or more retransmission timer timeout can occur during handoff. Due to the the timer backoff and coarse timer used in TCP, the communication can stop for a long time and cannot be resumed immediately even after the passing of the disconnection period until the retransmission timer expires. Shown in Fig. 1 is a segment-time graph of a TCP connection in response to disconnections.

Although the whole window of packets can be lost and timer can expire during handoff, it is also possible that only a portion of the packets in the current window are lost and timeout does not occur if handoff can complete reasonably fast. In the latter case, the packets in the later portion of the current window can reach the $\mathrm{MH}$ and trigger fast retransmit (which can eliminate the long post-handoff pause) at the TCP sender (FH) once their acknowledgments reach the FH. Unfortunately, the bulk losses ${ }^{2}$ occurring during handoff can cause multiple losses in a single window which make Reno-TCP lose its self-clocking property, whereas it is fine for Tahoe-TCP. The effects of this multiple losses can last even longer than the loss of the whole window of packets for Reno-TCP.

\section{B. Slow Post-handoff Recovery}

The congestion window cund drops to its minimum, 1 MSS (Maximum Segment Size), after a cell crossing (disconnection) as a result of one or more expirations of the retransmission timer during handoff and returns only gradually to its previous level once transport-level communication resumes. TCP's slow start algorithm [5], is responsible for this behavior. As acknowledgments are received, cund grows exponentially until the ssthresh (slow-start threshold) is reached, then it grows slowly and linearly. The slow-start threshold is set to one half of the congestion window size at the time of retransmission timeout. Consecutive timeouts during the disconnection cause ssthresh to decay exponentially. If retransmission timeout occurs several times which is common for disconnections with long duration (handoff crossing non-overlapping cells), the value of ssthresh will be unreasonably small causing slow recovery after the resumption of the transportlevel communication.

\footnotetext{
${ }^{2}$ Bulk losses during handoff are particularly serious to TCP since the TCP packets arrive in clusters[9].
}

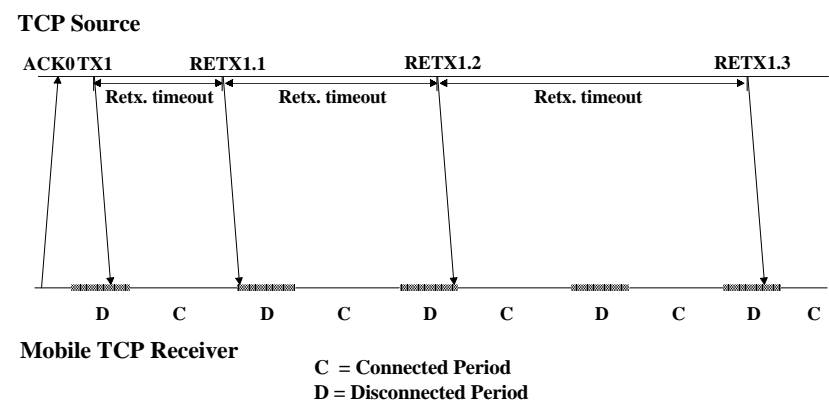

Fig. 2. Successive timeouts at the TCP sender.

\section{Successive Timeouts due to Handoff}

In order to provide high aggregate bandwidth, cell size will have to be reduced. Unfortunately, small cell sizes result in short cell resident time, which in turn, result in frequent disconnections as a user roams. All the problems that result from disconnections, discussed above, occur more often here.

Another problem caused by small cell resident time and frequent disconnections is successive timeouts at the TCP sender. A successive timeout (as shown in Fig. 2) is a condition wherein multiple consecutive retransmissions of the same TCP segment to the mobile take place while it is disconnected, not necessarily during the same disconnection. All these retransmissions are lost. Due to timer backoff for each unsuccessful retransmission attempt (until it reaches 64), several consecutive failures can lead to periods of inactivity lasting several minutes. Even when the mobile is reconnected, no data may be transmitted for as long as 1 minute. Besides, the values of cwnd and ssthresh are usually very small (1 MSS) after the communication resumes.

We have found that successive timeouts at the TCP sender can be even more harmful to the overall throughput than losses due to small congestion window size. Our simulation experiments also show that the probability of occurrences and the period of successive-timeout-caused inactivity increase as the cell resident time decreases or disconnected period duration increases. Our results show that the inactivity period longer than several disconnected periods starts to occur when the ratio of the disconnected period duration to the cell resident time is greater than 0.3. As an example, for a case with disconnected period duration of $2 \mathrm{~s}$ and cell resident time of $5 \mathrm{~s}$, the inactivity period occurs approximately once every 5 minutes and lasts for approximately 30s.

\section{ili. Alleviating the Effects of Motion}

To alleviate the problems caused by host motion, it is necessary to ensure that when the mobile is reconnected, it begins receiving data immediately rather than having to wait for the sender ${ }^{3}$ to timeout and retransmit. Furthermore, it is necessary to prevent the sender from shrinking its congestion window (cwnd) when packets are lost during the disconnection. To do these, two approaches are possible:

- Hiding motion from the transport level so that the TCP sender is unaware of disconnection.

- Making the TCP sender aware of the losses due to motion to distinguish these from the losses due to network congestion and adapting the transport level to react better to motion.

\footnotetext{
${ }^{3}$ In this paper, a connection from a fixed host (FH) to a mobile host $(\mathrm{MH})$ is considered since most of the problems occur in this configuration. For a connection from $\mathrm{MH}$ to $\mathrm{FH}$, the $\mathrm{MH}$ usually has the complete knowledge of the motion.
} 
In this section, two schemes proposed to alleviate the effects of motion, PROBE and BUFFER+FREEZE, are described. PROBE adapts the TCP sender to motion while BUFFER+FREEZE shields the TCP connection from the effects of motion.

\section{A. PROBE}

\section{A.1 Principle}

PROBE is adopted from the idea of network layer signaling in [2]. Fast Retransmit TCP (FR-TCP)[2] tries to solve the disconnection problem during handoff by using information from the Mobile IP to detect the completion of handoff. To resume transport layer communication earlier (without relying on timeout) and eliminate long idle time after handoff, (three) duplicate acknowledgments are intentionally sent to the TCP sender at FH to trigger fast retransmit once handoff completes. However, without using any new end-to-end TCP acknowledgment semantics (i.e. using new kind of acknowledgments to denote the end of handoff), the fast retransmit approach does not work with the recent versions of TCP in which the bug of false fast retransmit [4] is fixed. In the bug-fixed versions of TCP, if a timeout occurs, any duplicate acknowledgments within the same retransmission window (that is, from the packet with timeout to the highest packet sent when timeout occurs) will not be allowed to trigger fast retransmit. With the fast retransmit approach in [2], timeouts occurring during handoff will render the fast retransmit mechanism in the bug-fixed versions of TCP inactive with respect to the duplicate acknowledgments intentionally sent after handoff. Therefore, the approach in [2] does not function as usual if no new type of acknowledgments are used. In addition, FR-TCP does not solve the slow recovery problem after handoff.

The acknowledgment problem discussed above can be fixed using the negative acknowledgment discussed in [3]. Besides, in order to solve the slow recovery problem after handoff, a new algorithm is devised in PROBE to estimate the available capacity in the new radio cell and to detect missing packets. The purposes are to retransmit missing packets earlier and to open up the congestion window (cwnd) faster after the resumption of the TCP layer communication.

\section{A.2 Operation}

For a connection from FH to MH, after the completion of handoff, the network layer (IP) at the MH signals the TCP receiver at the $\mathrm{MH}$ this instance. In response, the TCP receiver sends three copies ${ }^{4}$ of negative acknowledgment (nack), special to denote handoff completion, to acknowledge the latest in-sequence packets received after handoff. Any one of the three nack received at the source will trigger a handoff recovery phase.

In the handoff recovery phase,the source first disables the fast retransmit mechanism to all the duplicate acknowledgments for the packets already sent. This is to avoid the loss of self-clocking due to multiple losses in a single window if only packets in the early portion of the previous transmission window are lost. The missing packets will be sent through the information in the bitmask fed back from the receiver. Then the TCP sender sends three ${ }^{5}$ consecutive packets (with different sequence numbers) just after the acknowledgment and with the option field set appropriately to request for receiver's buffer status. These probing packets serve two purposes:

\footnotetext{
${ }^{4}$ Two of the three nacks are sent as redundancy to have "time diversity" over wireless channels.

${ }^{5}$ Two packets will be sufficient, the extra one is redundant for reliability These packets should also be well protected by in-packet redundancy.
}

- Upon receiving any two consecutive packets, the receiver can estimate the available wireless bandwidth (assumed to be the bottleneck) as the inverse of the time interval elapsed between the arrivals of the two consecutive packets (e.g. average can be used for multiple measured values). This information is then fed back to the TCP sender. ${ }^{6}$

- Besides, these packets act as request packets to ask the TCP receiver to feedback its receiving buffer status, including the sequence number of highest in-sequence byte received and the highest sequence number of the byte received so far, followed by a list of the starting sequence number and the ending sequence number of the bytes missing at the TCP receiver. This is similar to the bitmask used in the RLP of US TDMA [1]. The extra traffic due to the bitmask is justified since only one bitmask packet will be sent for each handoff.

Upon receiving the bitmask packet, the estimated bandwidth together with the round-trip time measured are used to set the ssthresh accordingly. This is similar to the packet-pair probe used for congestion control in [6]. The cwnd is then set to half of the new value of ssthresh. These value settings solve the small window and slow recovery problem while avoid injecting too many packets into the connection over a new wireless link after handoff. The variable seqno (normally used in TCP) and a new variable recovery_end are set to the highest in-sequence segment number and the highest segment number received at the receiver respectively. The variable recovery_end is to signify the end of the handoff recovery phase. When an acknowledgment with sequence number greater than or equal to recovery_end is received, the source will exit the handoff recovery phase. The seqno at the TCP sender together with this recovery_end define the retransmission window.

Based on the bitmask, the TCP sender put all the missing data into a retransmission buffer. When the missing data are retransmitted, a variable count is incremented accordingly. The data in the retransmission buffer can be retransmitted as long as count is smaller than cund. A new variable highest_rsent will be set to the highest sequence number in the retransmission window retransmitted. Upon receiving an acknowledgment in the retransmission window, no matter it is new or duplicate, count is decremented while cwnd and ssthresh are adjusted as usual to avoid congestion in the new radio cell. Only the packet just after the duplicate acknowledgment and the packets after highest_rsent are sent if duplicate acknowledgments are received or timeouts occur for packets within the retransmission window. The packets in between are not sent immediately.

New data can be sent if they fall between last_ack (last in-sequence acknowledgment received at the TCP sender) and last_ack+cwnd-count. When an acknowledgment greater than or equal to recovery_end is received, the handoff recovery phase exits and normal TCP operation is resumed.

This procedure is the same for a connection from $\mathrm{MH}$ to $\mathrm{FH}$, except no nack is necessary to initiate the handoff recovery phase since this time the source ( $\mathrm{MH}$ ) has the exact knowledge about the handoff and can trigger handoff recovery phase as soon as the handoff complete signal from IP is received.

\footnotetext{
${ }^{6}$ Instead of measuring the inter-arrival time between the corresponding acknowledgments at the sender, the inter-arrival time between two consecutive packets are measured at the receiver and then fed-back to the sender The main reason of doing so is to avoid the effects of acknowledgment compression [9] which gives an inaccurate estimate. Besides, the noisy return channel causes a lower probability of receiving uncorrupted acknowledgments for two consecutive packets at the sender compared to receiving two uncorrupted and consecutive packets at the receiver.
} 


\section{B. BUFFER+FREEZE}

\section{B.1 Principle}

Buffering at the basestation seems to be able to eliminate packet losses during handoff and thus hide host motion from the TCP. However, there are two problems which make buffering infeasible:

- If the handoff takes a long time to complete (maybe due to long distance between two non-overlapping cells), even though there are no losses and the transport layer communication can be resumed immediately once the MH is reconnected, retransmission timeout can occur several times at the TCP sender. These timeouts can make the ssthresh very small or even equal to 1 MSS. Once the communication resumes, it still takes a lot of time for the TCP sender to resume the transmission rate to its previous level. If handoff occurs frequently, the throughput can suffer severely. Besides, the delay in buffering the packets can inflate the estimate of the timeout values (RTT, SRTT, RTO) which affect TCP's performance in a non-trivial manner.

- Buffering also increases the memory requirement of the BS. Although it can be proved that the amount of data that a BS needs to buffer is bounded by the maximum handoff latency between adjacent cells, this can be large if the handoff delay is large particularly for connection with a large wired bandwidth or round-trip delay.

To solve this problem, we propose to make TCP sender unaware of the occurrence of the motion by freezing all its retransmission timer and states. To do this, the $\mathrm{BS}$ is made to change the information in the TCP acknowledgments to make the sender interpret the event as a receiver's buffer depletion. This has several advantages:

- First, it freezes the cund, ssthresh and the retransmission timer at the TCP sender so that the related problems of long pauses in communication and slow recovery after handoff are avoided.

- Second, it freezes the activity of the TCP sender which prevents further data sending from the TCP sender. This can avoid using too much memory at the BS.

- Finally, this scheme can also work without buffering. The only difference is that the packets have to be retransmitted from the TCP sender.

\section{B.2 Operation}

The BS keeps track of the highest acknowledgment mss_hack received and buffers the subsequent packets in its cache. The BS also keeps a record of the highest sequence number sent mss_hseqno.

Acknowledgments received at the BS are normally buffered and kept at the BS before passing to the TCP sender at the FH. An exception is that when there are no outstanding unacknowledged packets or it is a duplicate acknowledgment, the acknowledgment will be passed to the sender immediately. The latest acknowledgment will be stored at the BS until it is replaced by a newly received acknowledgment or the local timer ${ }^{7}$ expires, then the acknowledgment will be passed to the sender. For the former case with replacement, the local timer is restarted if there are outstanding unacknowledged packets and no modifications to the header of the acknowledgment are made. In the latter, the acknowledgment is passed to the sender with the advertised window (snd_wnd) field reseted to 0 . In case of local timer timeout, it is suspected that a handoff or disconnection is in progress and the BS informs the

\footnotetext{
${ }^{7}$ The timeout value is based on the estimated round-trip time over the wireless link, similar to the estimation in TCP retransmission timeout
}

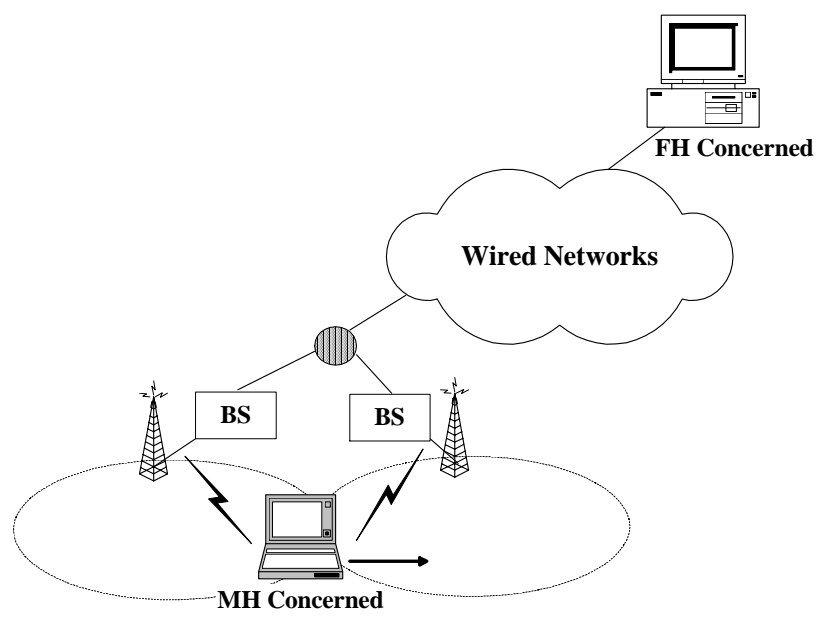

Fig. 3. The handoff simulation scenario

sender this event as a receiver's buffer outage to freeze its actions. The old BS itself enters the persist reply mode. Whenever a new packet arrives at $\mathrm{BS}$ from the $\mathrm{FH}$ or a new acknowledgment arrives from the $\mathrm{MH}$, the local timer is started if it has not been set previously.

Upon receiving the acknowledgment with 0 advertised window, the TCP sender enters the persist mode. In the persist mode, all states and retransmission timer at the sender are frozen and the sender periodically sends a segment to probe the receiver once the persist timer expires. In normal operation, the receiver will return its latest window size information in the acknowledgment. In PROBE, the old BS, which has entered the persist reply mode, will take up all the responsibility to reply to these persist segments by sending an acknowledgment with 0 advertised window if the handoff has not passed. Any acknowledgment from the MH to the old BS can take the old BS out of the persist reply mode, after that the old BS forwards acknowledgments as usual.

Once the handoff is completed, the old BS is notified by the new BS. The sender can come out from the persist mode if either an acknowledgment with the original advertised window value set is sent to the sender from either BS's or a new acknowledgment with the latest advertised window is sent from the $\mathrm{MH}$ upon receiving new packets after handoff.

\section{Performance Comparisons}

\section{A. Simulation Scenario}

The simulation scenario is depicted in Fig. 3. The MH moves between the two BS's. The time the MH spends in a cell is exponentially distributed with mean cell resident time $\gamma$ and the duration of the disconnected period during handoff is called the handoff time $h t$. The BS's in turn are connected to a FH via an error free wired connection with round-trip propagation delay of $60 \mathrm{~ms}$. The wireless links are assumed to be error free and have round-trip propagation delay of $2 \mathrm{~ms}$. Each wireless link has a capacity of $64 \mathrm{kbps}$ while the wired link has a capacity of $10 \mathrm{Mbps}$. 4.3 BSD Reno-TCP and Tahoe-TCP in the Network Simulator [7] are used for the simulations. The TCP connection performs a bulk file transfer using MSS equal to 512 bytes.

\section{B. Effects of Handoff Time}

Fig. 4 shows how the throughput of Reno-TCP and Tahoe-TCP are affected by the handoff time $h t$ for the mean cell resident time $\gamma=5,10,20 \mathrm{~s}$. Both PROBE and BUFFER+FREEZE offer 


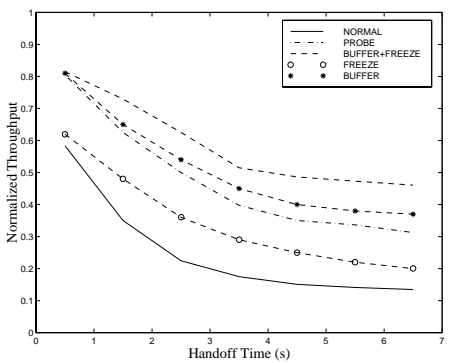

(a) Reno, $\gamma=5 \mathrm{~s}$

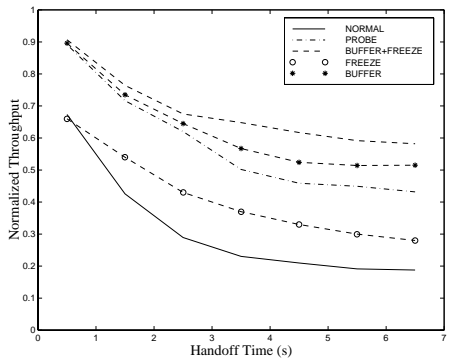

(b) Reno, $\gamma=10 \mathrm{~s}$

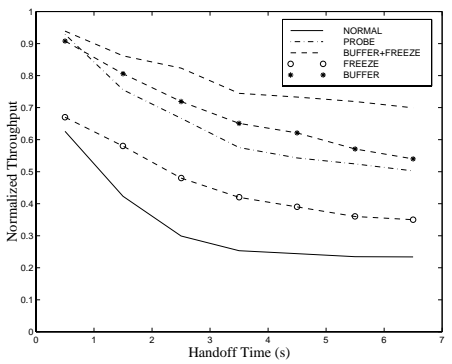

(c) Reno, $\gamma=20 \mathrm{~s}$

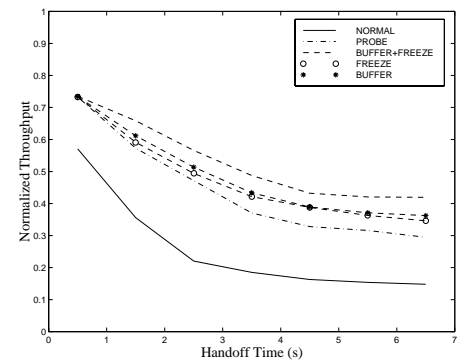

(d) Tahoe, $\gamma=5 \mathrm{~s}$

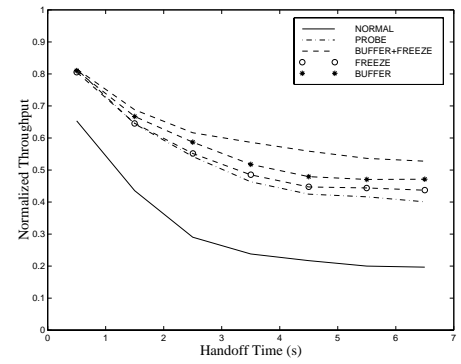

(e) Tahoe, $\gamma=10 \mathrm{~s}$

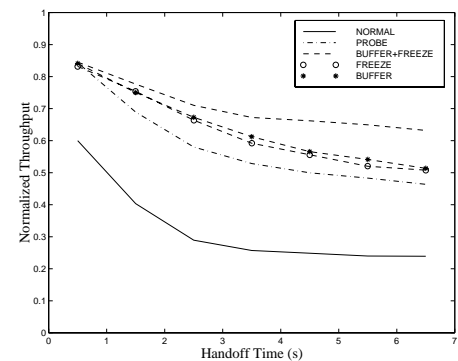

(f) Tahoe, $\gamma=20 \mathrm{~s}$
Fig. 4. The effects of handoff time $h t$ on the throughput of Reno-TCP and Tahoe-TCP.

significant throughput improvement relative to normal TCP for both Tahoe and Reno. BUFFER+FREEZE has a much better improvement than PROBE because no packets are lost during handoff and the buffered packets are retransmitted locally from the BS to the MH immediately after handoff completes. The local path has a much smaller propagation delay and hence offers faster recovery. As expected, as $h t$ increases, the throughput drops.

From the figures, it can be seen that if buffering is used without freezing (BUFFER), there are still significant improvement over normal TCP for both Tahoe and Reno. However, if the TCP source is frozen without buffering at the basestation (FREEZE), there are still small but not very significant improvement for the Reno-TCP. On the contrary, FREEZE still works quite well with Tahoe-TCP. It is mainly because Reno-TCP is not robust to multiple losses in a single window. Although FREEZE can freeze the variable such as cwnd, the bulk losses during handoff can still trigger cwnd reduction and make Reno-TCP lose its self-clocking property after handoff completes. After detecting the losses during handoff, Tahoe-TCP reduces cwnd to one, that is, it implicitly assumes all the packets after last_ack are lost and all these packets will be transmitted again. Tahoe-TCP is robust with respect to multiple losses in a single window.

\section{Effects of Cell Resident Time}

Fig. 5 shows how the Reno-TCP throughput is affected by the mean cell resident time $\gamma$ for handoff time $h t=1.5$ and 2.5s. As

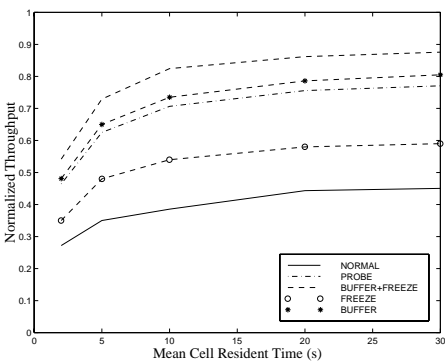

(a) Reno, $h t=1.5 \mathrm{~s}$

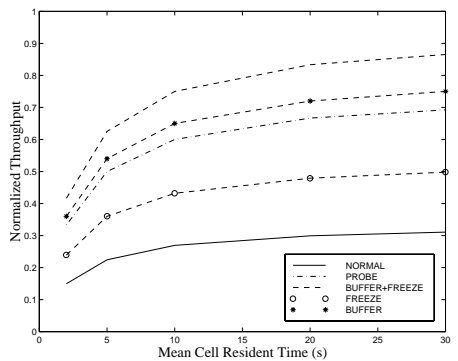

(b) Reno, $h t=2.5 \mathrm{~s}$
Fig. 5. The effects of mean cell resident time $\gamma$ on the throughput of Reno-TCP.

expected, as $\gamma$ increases, the throughput increases.

\section{Conclusion}

Since TCP is a very commonly used transport layer protocol over a wide variety of networks, the study of its implementation over the mobile cellular networks becomes an interesting research topic in mobile computing and is necessary for smooth and harmonized introduction of wireless/mobile data systems. In this paper, the causes for TCP throughput loss in the presence of handoff are investigated and three phenomena causing motion related throughput degradation, long communication pause, slow post-handoff recovery and successive retransmission, are discussed.

Two schemes, PROBE and BUFFER+FREEZE, are proposed to alleviate the effects of motion on TCP. PROBE adapts TCP to motion while BUFFER+FREEZE hides motion from TCP. BUFFER+FREEZE is found to have a better performance than PROBE. With such modified TCP, the use of many of the existing network software/programs such as "HTTP", "TELNET", and "FTP" can be extended to portable computers connected to the servers via mobile wireless links. This allows the use of the existing softwares in mobile computing environment and faster introduction of wireless date service. Besides, the schemes proposed in this paper can work well with the schemes proposed to deal with wireless link corruptions[3].

\section{REFERENCES}

[1] R. P. Zjzak, S. Nanda, and B. T. Doshi. "A retransmission scheme for circuit-mode data on wireless links". IEEE Journal on Selected Areas in Communications, 12(8):1338-1352, October 1994

[2] R. Cáceres, and L. Iftode. "Improving the performance of reliable transport protocols in mobile computing environments". IEEE Journal on Selected Areas in Communications, 13(5):850-857, June 1995

[3] A. C. F. Chan, D. H. K. Tsang, and S. Gupta. "TCP (Transmission Control Protocol) over Wireless Links". In Proceeding of VTC'g7, p. 2326-30, Phoenix, U.S.A., May 1997

[4] S. Floyd. "TCP and successive fast retransmits". Obtained via: "ftp://ftp.eellbl.gov/papers/fastretrans.ps", February, 1995

[5] V. Jacobson. "Congestion avoidance and control". ACM Computer Communication Review, 18(4):314-29, August 1988.

[6] S. Keshav. "A control-theoretic approach to flow control". ACM Computer Communication Review, 21(4):3-15, September 1991

[7] S. McCanne, S. Floyd, and K. Fall. "NS - LBNL Network Simulator" Obtained via: "http://www-nrg-eellbl.gov/ns"

[8] P Manzoni, D Ghosal, and G. Serazzi. "A simulation study of the impact of mobility on TCP/IP". IEEE Journal on Selected Areas in Communications, 13(5):858-67, June 1995

[9] L. Zhang, S. Shenker, and D. D. Clark. "Observations on the dynamics of a congestion control algorithm: The effects of two-way traffic" ACM Computer Communication Review, 21(4):133-147, September 1991.

[10] J. Postel "Transmission Control Protocol - DAPRA Internet Program Protocol Specification". RFC793, September 1981.

[11] "Webpage of the Metricom's Ricochet Networks" at "http://www ricochet net/ricochet" 